\title{
Effective Neutrino Masses from Four Flavor Neutrino Mixing Matrix
}

\author{
Bipin Singh Koranga ${ }^{1,2}$ (D) . Vivek Kumar Nautiyal ${ }^{1,2}$
}

Received: 7 October 2020 / Accepted: 3 December 2020 / Published online: 31 March 2021

(C) The Author(s) 2021

\begin{abstract}
We consider the four neutrino oscillation that accommodate the all neutrino oscillation data. We consider the range of the corresponding mixing parameters by the result of neutrino oscillation experiments. Implicaion of the neutrino oscillation search for the neutrino mass square difference and mixing are discussed. We determine the possible values of the effective majorana neutrino mass $|<m>|=\left|\sum_{j} U_{e j}^{2} m_{j}\right|$ in the four neutrino scenario. In the four-neutrino scheme there is an upper bound on $|<m>|$ of the normal mass order is $2.0074 \mathrm{eV}$ for $\alpha=0^{\circ}, \beta=0^{\circ}$ and $\gamma=0^{\circ}$. In the case of inverted mass order the upper bound on $|<\mathrm{m}>|$ is $2.0069 \mathrm{eV}$ for $\alpha=0^{\circ}, \beta=0^{\circ}$ and $\gamma=0^{\circ}$.
\end{abstract}

Keywords Neutrino masses · Four flavor neutrino mixing

\section{Introduction}

The phenomenon of neutrino oscillation, impressive advance have been made to understand the phenomenology of neutrino oscillation through solar neutrino,atmospheric neutrino,reactor neutrino and accelerator neutrino experiment. The experimental research on the nature of neutrinos through terrestrial as well as extra-terrestrial approaches has finally confirmed the neutrino oscillation in atmospheric [1-7] solar [8-14], reactor [15-17] and accelerator $[18,19]$ neutrino sources, establishing that neutrinos have mass. Furthermore, it is generally agreed that oscillations among three neutrino species are sufficient to explain the atmospheric, solar, reactor and accelerator neutrino puzzle. The neutrino oscillation experiments provide us with neutrino mass square differences, mixing angles and a possible hierarchy in the neutrino mass spectrum. The main physical goal in future experiment are the determination of the unknown parameter $\theta_{13}$. In particular, the observation of $\delta$ is

Bipin Singh Koranga

bipiniitb@rediffmail.com

1 Department of Physics, Kirori Mal College (University of Delhi), Delhi, 110007, India

2 Department of Physics, Babasaheb Bhimrao Ambedkar University, Lucknow, 226025, India 
quite interesting from the point of view that $\delta$ is related to the origin of the matter in the universe. One of the most important parameter in neutrino physics is the magnitude of mixing angle $\theta_{13}$ and $\mathrm{CP}$ phase $\delta$. The oscillation data also suggests that the neutrinos may belong to either a normal hierarchy $\left(m_{1}<m_{2}<m_{3}\right)$ or an inverted hierarchy $\left(m_{3}<m_{1}<m_{2}\right)$. The data do not exclude the possibility that the mass of the light neutrino could be much larger than $\sqrt{\Delta_{31}}$, which would imply the possible existence of a quasi-degenerate neutrino mass spectrum $\left(m_{1} \approx m_{2} \approx m_{3}\right)$. On the other hand, the actual mass of neutrinos cannot be extracted from these data, only the study of tritium single $\beta$ decay and nuclear double beta decay together can provide sharpest limits on the mass and nature of neutrinos. Neutrino oscillations, which only depend on mass square difference, give no information about the absolute value of the neutrino mass squared eigenvalues. Hence, there are various possibilities of neutrino hierarchy spectrums consistent with solar and atmospheric neutrino oscillation data. The mass eigenstates with eigen values $m_{i}$ can be determined if the absolute value of effective mass of neutrino is exactly known. The current neutrinoless double beta decay experiments only provide the upper limit on effective Majorana neutrino mass $<m_{e e}>$ so that absolute scale of neutrino mass is not determined yet. Therefore, in the

Table 1 For $\alpha=0^{\circ}$ and normal mass order, the effective Neutrino Mass for different value of Majorona phases

\begin{tabular}{lll}
\hline$\beta$ & $\gamma$ & $m_{e e}(\mathrm{eV})$ \\
\hline $0^{\circ}$ & $0^{\circ}$ & 2.0074 \\
$0^{\circ}$ & $45^{\circ}$ & 1.9944 \\
$0^{\circ}$ & $90^{\circ}$ & 1.9626 \\
$0^{\circ}$ & $135^{\circ}$ & 1.9301 \\
$0^{\circ}$ & $180^{\circ}$ & 1.9167 \\
$45^{\circ}$ & $0^{\circ}$ & 1.9905 \\
$45^{\circ}$ & $45^{\circ}$ & 1.9781 \\
$45^{\circ}$ & $90^{\circ}$ & 1.9467 \\
$45^{\circ}$ & $135^{\circ}$ & 1.9141 \\
$45^{\circ}$ & $180^{\circ}$ & 1.8998 \\
$90^{\circ}$ & $0^{\circ}$ & 1.9491 \\
$90^{\circ}$ & $45^{\circ}$ & 1.9371 \\
$90^{\circ}$ & $90^{\circ}$ & 1.9057 \\
$90^{\circ}$ & $135^{\circ}$ & 1.8730 \\
$90^{\circ}$ & $180^{\circ}$ & 1.8585 \\
$135^{\circ}$ & $0^{\circ}$ & 1.9068 \\
$135^{\circ}$ & $45^{\circ}$ & 1.8945 \\
$135^{\circ}$ & $90^{\circ}$ & 1.8631 \\
$135^{\circ}$ & $135^{\circ}$ & 1.8305 \\
$135^{\circ}$ & $180^{\circ}$ & 1.8162 \\
$180^{\circ}$ & $0^{\circ}$ & 1.8890 \\
$180^{\circ}$ & $45^{\circ}$ & 1.8760 \\
$180^{\circ}$ & $90^{\circ}$ & 1.8442 \\
$180^{\circ}$ & $135^{\circ}$ & 1.8119 \\
$180^{\circ}$ & $180^{\circ}$ & 1.7984 \\
\hline & & \\
\hline & &
\end{tabular}


present work we have attempted to present a effective neutrino neutrino mass in the case of normal and inverted neutrino mass order.

In this paper, we will discuss the effective neutrino masses for different neutrino mass spectrum, namely normal mass hierarchy $\left(m_{1}<m_{2}<m_{3}\right)$, inverted mass hierarchy $\left(m_{3}<m_{1}<m_{2}\right)$. The present work is organized as follows. In Section 2, we outline the neutrino oscillation parameters. In Section 3, we have given the theoretical formalism to calculate the effective neutrino masses above mentioned hierarchies. In Section 4, we present the numerical results and Section 5 is devoted to the conclusions.

\section{Mixing Angles and Neutrino Mass Squared Differences}

The first evidence is the observation of zenith-angle dependence of atmospheric neutrino defect [20] dependent of the atmospheric neutrino $v_{\mu} \rightarrow v_{\mu}$ transition with the mass difference and the mixing as

$$
\Delta_{31}=(1-2) \times 10^{-3} e V^{2}, \sin ^{2} 2 \theta_{23}=1.0 .
$$

Table 2 For $\alpha=45^{\circ}$ and normal mass order, the effective Neutrino Mass for different value of Majorona phases

\begin{tabular}{lll}
\hline$\beta$ & $\gamma$ & $m_{e e}(\mathrm{eV})$ \\
\hline $0^{\circ}$ & $0^{\circ}$ & 1.8808 \\
$0^{\circ}$ & $45^{\circ}$ & 1.8753 \\
$0^{\circ}$ & $90^{\circ}$ & 1.8475 \\
$0^{\circ}$ & $135^{\circ}$ & 1.8132 \\
$0^{\circ}$ & $180^{\circ}$ & 1.7925 \\
$45^{\circ}$ & $0^{\circ}$ & 1.8738 \\
$45^{\circ}$ & $45^{\circ}$ & 1.8691 \\
$45^{\circ}$ & $90^{\circ}$ & 1.8418 \\
$45^{\circ}$ & $135^{\circ}$ & 1.8074 \\
$45^{\circ}$ & $180^{\circ}$ & 1.7860 \\
$90^{\circ}$ & $0^{\circ}$ & 1.8377 \\
$90^{\circ}$ & $45^{\circ}$ & 1.8336 \\
$90^{\circ}$ & $90^{\circ}$ & 1.8066 \\
$90^{\circ}$ & $135^{\circ}$ & 1.7721 \\
$90^{\circ}$ & $180^{\circ}$ & 1.7503 \\
$135^{\circ}$ & $0^{\circ}$ & 1.7928 \\
$135^{\circ}$ & $45^{\circ}$ & 1.7886 \\
$135^{\circ}$ & $90^{\circ}$ & 1.7616 \\
$135^{\circ}$ & $135^{\circ}$ & 1.7270 \\
$135^{\circ}$ & $180^{\circ}$ & 1.7054 \\
$180^{\circ}$ & $0^{\circ}$ & 1.7656 \\
$180^{\circ}$ & $45^{\circ}$ & 1.7607 \\
$180^{\circ}$ & $90^{\circ}$ & 1.7333 \\
$180^{\circ}$ & $135^{\circ}$ & 1.6988 \\
$180^{\circ}$ & $180^{\circ}$ & 1.6777 \\
\hline & & \\
& &
\end{tabular}


From the recent three neutrino analysis of the Super-Kamiokande data [29] the following $90 \% \mathrm{CL}$ were found for the normal (inverted) neutrino mass spectrum

$$
1.9(1.7) \times 10^{-3} e V^{2} \leq \Delta_{31} \leq 2.6(2.7) \times 10^{-3} e V^{2}
$$

The second evidence is the solar neutrino deficit [21], which is consistent with $v_{\mu} \rightarrow$ $v_{\tau} / v_{e}$ transition. The SNO experiments [22] are consistent with the standard solar model [23] and strongly suggest the LMA solution.

$$
\Delta_{21}=7 \times 10^{-5} e V^{2}, \sin ^{2} 2 \theta_{12}=0.8 .
$$

Solar neutrino experiments (Super-K, GALLEX, SAGE, SNO and GNO) show the neutrino oscillations, neutrino oscillation provide the most elegant explanation of all the data [24].

$$
\begin{gathered}
\Delta_{\text {solar }}=7_{-1.3}^{+5} \times 10^{-5} \mathrm{eV}^{2}, \\
\tan ^{2} \theta_{\text {solar }}=0.4_{-0.1}^{+0.14} .
\end{gathered}
$$

From the three neutrino global analysis of the solar and reactor KamLAND data was found [27],

$$
\Delta_{21}=7.5_{-.20}^{+.19} \times 10^{-3} e V^{2}, \quad \tan ^{2} \theta_{12}=0.452_{-0.032}^{+0.035}
$$

Table 3 For $\alpha=90^{\circ}$ and normal mass order, the effective Neutrino Mass for different value of Majorona phases

\begin{tabular}{lll}
\hline$\beta$ & $\gamma$ & $m_{e e}(\mathrm{eV})$ \\
\hline $0^{\circ}$ & $0^{\circ}$ & 1.5326 \\
$0^{\circ}$ & $45^{\circ}$ & 1.5332 \\
$0^{\circ}$ & $90^{\circ}$ & 1.5096 \\
$0^{\circ}$ & $135^{\circ}$ & 1.4749 \\
$0^{\circ}$ & $180^{\circ}$ & 1.4495 \\
$45^{\circ}$ & $0^{\circ}$ & 1.5335 \\
$45^{\circ}$ & $45^{\circ}$ & 1.5351 \\
$45^{\circ}$ & $90^{\circ}$ & 1.5123 \\
$45^{\circ}$ & $135^{\circ}$ & 1.4777 \\
$45^{\circ}$ & $180^{\circ}$ & 1.4515 \\
$90^{\circ}$ & $0^{\circ}$ & 1.5030 \\
$90^{\circ}$ & $45^{\circ}$ & 1.5054 \\
$90^{\circ}$ & $90^{\circ}$ & 1.4832 \\
$90^{\circ}$ & $135^{\circ}$ & 1.4487 \\
$90^{\circ}$ & $180^{\circ}$ & 1.4219 \\
$135^{\circ}$ & $0^{\circ}$ & 1.4578 \\
$135^{\circ}$ & $45^{\circ}$ & 1.4603 \\
$135^{\circ}$ & $90^{\circ}$ & 1.4381 \\
$135^{\circ}$ & $135^{\circ}$ & 1.4036 \\
$135^{\circ}$ & $180^{\circ}$ & 1.3768 \\
$180^{\circ}$ & $0^{\circ}$ & 1.4242 \\
$180^{\circ}$ & $45^{\circ}$ & 1.4260 \\
$180^{\circ}$ & $90^{\circ}$ & 1.4033 \\
$180^{\circ}$ & $135^{\circ}$ & 1.3687 \\
$180^{\circ}$ & $180^{\circ}$ & 1.3424 \\
\hline & & \\
& &
\end{tabular}


Atmospheric neutrino experiments (Kamiokande, Super-K) also show the neutrino oscillation. The most excellent fit to the all data [24].

$$
\begin{gathered}
\Delta_{\text {atmo }}=2.0_{-0.92}^{+1.0} \times 10^{-3} \mathrm{eV}^{2}, \\
\sin ^{2} 2 \theta_{\text {atmo }}=0.4_{-0.10}^{+0.14} .
\end{gathered}
$$

The CHOOZ reactor experiment [25] gives the upper bound on the third mixing angle $\theta_{13}$ as

$$
\begin{array}{llll}
\sin ^{2} \theta_{13}<0.20 & \text { for } & & \left|\Delta_{31}\right|=2.0 \times 10^{-3} e V^{2}, \\
\sin ^{2} \theta_{13}<0.16 & \text { for } & \left|\Delta_{31}\right|=2.5 \times 10^{-3} e V^{2}, \\
\sin ^{2} \theta_{13}<0.14 & \text { for } & \left|\Delta_{31}\right|=3.0 \times 10^{-3} e V^{2},
\end{array}
$$

at the $90 \% \mathrm{CL}$. The CP phase $\delta$ has not been constrained. From the two neutrino analysis of the MINOS data obtained was [26],

$$
\sin ^{2} \theta_{13}<0.90 \quad \text { for } \quad\left|\Delta_{31}\right|=(2.43 \pm 0.13) \times 10^{-3} e V^{2},
$$

The future neutrino experiments plan to measure the oscillation parameters precisely. The combined analysis of Day Bay, MINOS and Bugey-3 data [28] has excluded most

Table 4 For $\alpha=135^{\circ}$ and normal mass order, the effective Neutrino Mass for different value of Majorona phases

\begin{tabular}{lll}
\hline$\beta$ & $\gamma$ & $m_{e e}(\mathrm{eV})$ \\
\hline $0^{\circ}$ & $0^{\circ}$ & 1.0772 \\
$0^{\circ}$ & $45^{\circ}$ & 1.0780 \\
$0^{\circ}$ & $90^{\circ}$ & 1.0548 \\
$0^{\circ}$ & $135^{\circ}$ & 1.0202 \\
$0^{\circ}$ & $180^{\circ}$ & 0.9943 \\
$45^{\circ}$ & $0^{\circ}$ & 1.0785 \\
$45^{\circ}$ & $45^{\circ}$ & 1.0808 \\
$45^{\circ}$ & $90^{\circ}$ & 1.0587 \\
$45^{\circ}$ & $135^{\circ}$ & 1.0242 \\
$45^{\circ}$ & $180^{\circ}$ & 0.9974 \\
$90^{\circ}$ & $0^{\circ}$ & 1.0487 \\
$90^{\circ}$ & $45^{\circ}$ & 1.0521 \\
$90^{\circ}$ & $90^{\circ}$ & 1.0309 \\
$90^{\circ}$ & $135^{\circ}$ & 0.9967 \\
$90^{\circ}$ & $180^{\circ}$ & 0.9690 \\
$135^{\circ}$ & $0^{\circ}$ & 1.0036 \\
$135^{\circ}$ & $45^{\circ}$ & 1.0071 \\
$135^{\circ}$ & $90^{\circ}$ & 0.9861 \\
$135^{\circ}$ & $135^{\circ}$ & 0.9518 \\
$135^{\circ}$ & $180^{\circ}$ & 0.9240 \\
$180^{\circ}$ & $0^{\circ}$ & 0.9693 \\
$180^{\circ}$ & $45^{\circ}$ & 0.9719 \\
$180^{\circ}$ & $90^{\circ}$ & 0.9501 \\
$180^{\circ}$ & $135^{\circ}$ & 0.9157 \\
$180^{\circ}$ & $180^{\circ}$ & 0.8885 \\
\hline & & \\
& &
\end{tabular}


Table 5 For $\alpha=180^{\circ}$ and normal mass order, the effective Neutrino Mass for different value of Majorona phases

\begin{tabular}{lll}
\hline$\beta$ & $\gamma$ & $m_{e e}(\mathrm{eV})$ \\
\hline $0^{\circ}$ & $0^{\circ}$ & 0.8173 \\
$0^{\circ}$ & $45^{\circ}$ & 0.8047 \\
$0^{\circ}$ & $90^{\circ}$ & 0.7733 \\
$0^{\circ}$ & $135^{\circ}$ & 0.74064 \\
$0^{\circ}$ & $180^{\circ}$ & 0.7267 \\
$45^{\circ}$ & $0^{\circ}$ & 0.8011 \\
$45^{\circ}$ & $45^{\circ}$ & 0.7902 \\
$45^{\circ}$ & $90^{\circ}$ & 0.7597 \\
$45^{\circ}$ & $135^{\circ}$ & 0.7264 \\
$45^{\circ}$ & $180^{\circ}$ & 0.7106 \\
$90^{\circ}$ & $0^{\circ}$ & 0.7604 \\
$90^{\circ}$ & $45^{\circ}$ & 0.7504 \\
$90^{\circ}$ & $90^{\circ}$ & 0.7204 \\
$90^{\circ}$ & $135^{\circ}$ & 0.6868 \\
$90^{\circ}$ & $180^{\circ}$ & 0.6701 \\
$135^{\circ}$ & $0^{\circ}$ & 0.7175 \\
$135^{\circ}$ & $45^{\circ}$ & 0.7069 \\
$135^{\circ}$ & $90^{\circ}$ & 0.6766 \\
$135^{\circ}$ & $135^{\circ}$ & 0.6432 \\
$135^{\circ}$ & $180^{\circ}$ & 0.6270 \\
$180^{\circ}$ & $0^{\circ}$ & 0.6990 \\
$180^{\circ}$ & $45^{\circ}$ & 0.6864 \\
$180^{\circ}$ & $90^{\circ}$ & 0.6552 \\
$180^{\circ}$ & $135^{\circ}$ & 0.6224 \\
$180^{\circ}$ & $180^{\circ}$ & 0.6083 \\
\hline & & \\
& & \\
\hline
\end{tabular}

of the parameter space of the mass squared difference $\Delta_{41}=m_{4}^{2}-m_{i}^{2}$ and the mixing angle $\sin ^{2} \theta_{14}$ for the sterile neutrino, the latest global analysis of neutrino oscillation data indicates that a small region arround the best-fit value of [29]

$$
\Delta_{41}=1.7 e V^{2} . \quad \text { and } \quad \sin ^{2} \theta_{14}=0.019
$$

\section{Effective Majorona Mass of Electron Neutrino}

In the presence of one sterile neutrino, in four flavour neutrino mixing the electron neutrino is combination of mass eigenstate, $v_{i}$ with eigenvalue $m_{i}$

$$
v_{e}=\sum U_{e j} v_{i j} \quad i=1,2,3,4
$$

Here $U_{e i}$ are the elements of the $4 \times 4$ mixing matrix, which relates the flavour states the the mass eigenstates. The $\beta \beta_{0 v}$ decay rate is determined by the effective Majorana mass of 
the electron neutrino $m_{e e}$. Under the assumption of four flavour neutrino mixing of neutrino, the effective Majorana neutrino mass $m_{e e}[30]$ is

$$
\begin{gathered}
\left|m_{e e}\right|_{4 v}=\left.\left|\sum\right| U_{e j}\right|^{2} e^{i \phi_{j}} m_{j} \mid \\
=\left|c_{13}^{2} c_{12}^{2} c_{14}^{2} m_{1}+c_{13}^{2} s_{12}^{2} c_{14}^{2} e^{i \alpha} m_{2}+s_{13}^{2} c_{14}^{2} e^{i \beta} m_{3}+s_{14}^{2} e^{i \gamma} m_{4}\right| .
\end{gathered}
$$

We consider that the sterile neutrino with mass $m_{4}$ is heavier than light active neutrinos. Therefore, the effective Majorana mass in case of normal ordering of active neutrino is given as

$m_{e e}=\left|c_{13}^{2} c_{12}^{2} c_{14}^{2} m_{1}+c_{13}^{2} s_{12}^{2} c_{14}^{2} e^{i \alpha} \sqrt{m_{1}^{2}+\Delta_{21}}+s_{13}^{2} c_{14}^{2} e^{i \beta} \sqrt{m_{1}^{2}+\Delta_{31}}+s_{14}^{2} e^{i \gamma} \sqrt{m_{1}^{2}+\Delta_{41}}\right|$.

Similarly for the case of inverted hierarchy of active neutrinos, the expression in (15) can be rewritten as

$$
\begin{aligned}
m_{e e}=\mid c_{13}^{2} c_{12}^{2} c_{14}^{2} \sqrt{m_{3}^{2}+\Delta_{23}-\Delta_{21}} & +c_{13}^{2} s_{12}^{2} c_{14}^{2} e^{i \alpha} \sqrt{m_{3}^{2}+\Delta_{23}} \\
& +s_{13}^{2} c_{14}^{2} e^{i \beta} m_{3}+s_{14}^{2} e^{i \gamma} \sqrt{m_{3}^{2}+\Delta_{43}} \mid .
\end{aligned}
$$

Table 6 For $\alpha=0^{\circ}$ and inverted mass order, the effective

Neutrino Mass for different value of Majorona phases

\begin{tabular}{lll}
\hline$\beta$ & $\gamma$ & $m_{e e}(\mathrm{eV})$ \\
\hline $0^{\circ}$ & $0^{\circ}$ & 2.0069 \\
$0^{\circ}$ & $45^{\circ}$ & 1.9939 \\
$0^{\circ}$ & $90^{\circ}$ & 1.9621 \\
$0^{\circ}$ & $135^{\circ}$ & 1.9298 \\
$0^{\circ}$ & $180^{\circ}$ & 1.9162 \\
$45^{\circ}$ & $0^{\circ}$ & 1.9899 \\
$45^{\circ}$ & $45^{\circ}$ & 1.9777 \\
$45^{\circ}$ & $90^{\circ}$ & 1.9462 \\
$45^{\circ}$ & $135^{\circ}$ & 1.9136 \\
$45^{\circ}$ & $180^{\circ}$ & 1.8994 \\
$90^{\circ}$ & $0^{\circ}$ & 1.9486 \\
$90^{\circ}$ & $45^{\circ}$ & 1.9366 \\
$90^{\circ}$ & $90^{\circ}$ & 1.9053 \\
$90^{\circ}$ & $135^{\circ}$ & 1.8726 \\
$90^{\circ}$ & $180^{\circ}$ & 1.8580 \\
$135^{\circ}$ & $0^{\circ}$ & 1.9063 \\
$135^{\circ}$ & $45^{\circ}$ & 1.8940 \\
$135^{\circ}$ & $90^{\circ}$ & 1.8626 \\
$135^{\circ}$ & $135^{\circ}$ & 1.8300 \\
$135^{\circ}$ & $180^{\circ}$ & 1.8157 \\
$180^{\circ}$ & $0^{\circ}$ & 1.8885 \\
$180^{\circ}$ & $45^{\circ}$ & 1.8755 \\
$180^{\circ}$ & $90^{\circ}$ & 1.8438 \\
$180^{\circ}$ & $135^{\circ}$ & 1.8115 \\
$180^{\circ}$ & $180^{\circ}$ & 1.7979 \\
\hline & & \\
\hline & & \\
\hline
\end{tabular}


Table 7 For $\alpha=45^{\circ}$ and inverted mass order, the effective Neutrino Mass for different value of Majorona phases

\begin{tabular}{lll}
\hline$\beta$ & $\gamma$ & $m_{e e}(\mathrm{eV})$ \\
\hline $0^{\circ}$ & $0^{\circ}$ & 1.8803 \\
$0^{\circ}$ & $45^{\circ}$ & 1.8748 \\
$0^{\circ}$ & $90^{\circ}$ & 1.8471 \\
$0^{\circ}$ & $135^{\circ}$ & 1.8127 \\
$0^{\circ}$ & $180^{\circ}$ & 1.7921 \\
$45^{\circ}$ & $0^{\circ}$ & 1.8733 \\
$45^{\circ}$ & $45^{\circ}$ & 1.8687 \\
$45^{\circ}$ & $90^{\circ}$ & 1.8414 \\
$45^{\circ}$ & $135^{\circ}$ & 1.8069 \\
$45^{\circ}$ & $180^{\circ}$ & 1.7856 \\
$90^{\circ}$ & $0^{\circ}$ & 1.8372 \\
$90^{\circ}$ & $45^{\circ}$ & 1.8331 \\
$90^{\circ}$ & $90^{\circ}$ & 1.8062 \\
$90^{\circ}$ & $135^{\circ}$ & 1.7717 \\
$90^{\circ}$ & $180^{\circ}$ & 1.7499 \\
$135^{\circ}$ & $0^{\circ}$ & 1.7923 \\
$135^{\circ}$ & $45^{\circ}$ & 1.7881 \\
$135^{\circ}$ & $90^{\circ}$ & 1.7611 \\
$135^{\circ}$ & $135^{\circ}$ & 1.7266 \\
$135^{\circ}$ & $180^{\circ}$ & 1.7049 \\
$180^{\circ}$ & $0^{\circ}$ & 1.7652 \\
$180^{\circ}$ & $45^{\circ}$ & 1.7602 \\
$180^{\circ}$ & $90^{\circ}$ & 1.7328 \\
$180^{\circ}$ & $135^{\circ}$ & 1.6984 \\
$180^{\circ}$ & $180^{\circ}$ & 1.6773 \\
\hline & & \\
\hline & & \\
\hline
\end{tabular}

The PMNS matrix $U_{4 \times 4}$ depends on the mixing angles and $\mathrm{CP}$ violating phases. Using the parameterization in (13)

$$
U=R_{34} R_{24} R_{14} R_{23} R_{13} R_{12} P,
$$

where the matrices $R_{i j}$ are rotations in ij space,

$$
R_{14}=\left(\begin{array}{cccc}
c_{14} & 0 & 0 & s_{14} e^{-i \delta_{14}} \\
0 & 1 & 0 & 0 \\
0 & 0 & 1 & 0 \\
-s_{14} e^{i \delta_{14}} & 0 & 0 & c_{14}
\end{array}\right) \quad \text { and } \quad R_{34}=\left(\begin{array}{cccc}
1 & 0 & 0 & 0 \\
0 & 1 & 0 & 0 \\
0 & 0 & c_{34} & s_{34} \\
0 & 0 & -s_{34} & c_{34}
\end{array}\right)
$$


Table 8 For $\alpha=90^{\circ}$ and inverted mass order, the effective Neutrino Mass for different value of Majorona phases

\begin{tabular}{lll}
\hline$\beta$ & $\gamma$ & $m_{e e}(\mathrm{eV})$ \\
\hline $0^{\circ}$ & $0^{\circ}$ & 1.5322 \\
$0^{\circ}$ & $45^{\circ}$ & 1.5328 \\
$0^{\circ}$ & $90^{\circ}$ & 1.5092 \\
$0^{\circ}$ & $135^{\circ}$ & 1.4745 \\
$0^{\circ}$ & $180^{\circ}$ & 1.4491 \\
$45^{\circ}$ & $0^{\circ}$ & 1.5331 \\
$45^{\circ}$ & $45^{\circ}$ & 1.5348 \\
$45^{\circ}$ & $90^{\circ}$ & 1.5119 \\
$45^{\circ}$ & $135^{\circ}$ & 1.4773 \\
$45^{\circ}$ & $180^{\circ}$ & 1.4512 \\
$90^{\circ}$ & $0^{\circ}$ & 1.5026 \\
$90^{\circ}$ & $45^{\circ}$ & 1.5050 \\
$90^{\circ}$ & $90^{\circ}$ & 1.4828 \\
$90^{\circ}$ & $135^{\circ}$ & 1.4483 \\
$90^{\circ}$ & $180^{\circ}$ & 1.4216 \\
$135^{\circ}$ & $0^{\circ}$ & 1.4574 \\
$135^{\circ}$ & $45^{\circ}$ & 1.4599 \\
$135^{\circ}$ & $90^{\circ}$ & 1.4377 \\
$135^{\circ}$ & $135^{\circ}$ & 1.4032 \\
$135^{\circ}$ & $180^{\circ}$ & 1.3765 \\
$180^{\circ}$ & $0^{\circ}$ & 1.4239 \\
$180^{\circ}$ & $45^{\circ}$ & 1.4256 \\
$180^{\circ}$ & $90^{\circ}$ & 1.4029 \\
$180^{\circ}$ & $135^{\circ}$ & 1.3684 \\
$180^{\circ}$ & $180^{\circ}$ & 1.3421 \\
\hline & & \\
\hline & & \\
& &
\end{tabular}

where $s_{i j}=\sin \theta_{i j}, c_{i j}=\cos \theta_{i j}$. The diagonal matrix $\mathrm{P}$ contains the three Majorana phases $\alpha, \beta$ and $\gamma$ :

$$
P=\operatorname{diag}\left(1, e^{i \alpha / 2}, e^{i\left(\beta+\delta_{13}\right)}, e^{i\left(\gamma / 2+\delta_{14}\right)}\right)
$$

Note that there are in total three Dirac CP-violating phase $\delta_{i j}$. P is constructed in such a way that only Majorana phases show up in the effective mass expression.

\section{Numerical Results}

Note from (16) and (17) that the effective neutrino depends on new physics involving sterile neutrino mixing angle $\theta_{14}$ and the mass square difference $\Delta_{41}$. Also cruically the type of neutrino mass spectrum, normal or inverse hierarchical spectrum. Hence, we consider a degenerated neutrino Mass spectrum and take the common neutrino mass to be $2 \mathrm{eV}$, which is upper limit of tritium beta decay spectrum [31]. 
Table 9 For $\alpha=135^{\circ}$ and inversted mass order, the effective Neutrino Mass for different value of Majorona phases

\begin{tabular}{lll}
\hline$\beta$ & $\gamma$ & $m_{e e}(\mathrm{eV})$ \\
\hline $0^{\circ}$ & $0^{\circ}$ & 1.0769 \\
$0^{\circ}$ & $45^{\circ}$ & 1.0778 \\
$0^{\circ}$ & $90^{\circ}$ & 1.0546 \\
$0^{\circ}$ & $135^{\circ}$ & 1.012 \\
$0^{\circ}$ & $180^{\circ}$ & 0.9941 \\
$45^{\circ}$ & $0^{\circ}$ & 1.0782 \\
$45^{\circ}$ & $45^{\circ}$ & 1.0806 \\
$45^{\circ}$ & $90^{\circ}$ & 1.0585 \\
$45^{\circ}$ & $135^{\circ}$ & 1.0240 \\
$45^{\circ}$ & $180^{\circ}$ & 0.9971 \\
$90^{\circ}$ & $0^{\circ}$ & 1.0484 \\
$90^{\circ}$ & $45^{\circ}$ & 1.0519 \\
$90^{\circ}$ & $90^{\circ}$ & 1.0307 \\
$90^{\circ}$ & $135^{\circ}$ & 0.9963 \\
$90^{\circ}$ & $180^{\circ}$ & 0.9687 \\
$135^{\circ}$ & $0^{\circ}$ & 1.0033 \\
$135^{\circ}$ & $45^{\circ}$ & 1.0069 \\
$135^{\circ}$ & $90^{\circ}$ & 0.9859 \\
$135^{\circ}$ & $135^{\circ}$ & 0.9516 \\
$135^{\circ}$ & $180^{\circ}$ & 0.9238 \\
$180^{\circ}$ & $0^{\circ}$ & 0.9691 \\
$180^{\circ}$ & $45^{\circ}$ & 0.9716 \\
$180^{\circ}$ & $90^{\circ}$ & 0.9498 \\
$180^{\circ}$ & $135^{\circ}$ & 0.9154 \\
$180^{\circ}$ & $180^{\circ}$ & 0.8883 \\
\hline & & \\
& & \\
\hline
\end{tabular}

In Tables 1, 2, 3, 4 and 5 for normal mass order and Tables 6, 7, 8, 9 and 10 for inverted mass order, we list the effective neutrino mass Vs majorana phases for CP phase conserved case. Using the best fit value [24-26, 29] of oscillation parameter. For the computational purpose, the Majorana phase are taken as $\alpha, \beta$ and $\gamma$. We have taken these values from $0-180$ degrees.

\section{Conclusions}

Future and present search for neutrinoless double beta decay purpose at probing lepton number violation and the Majorona nature of neutrinos with remarkable precession. Several experimental programs is currently under discussion. 
Table 10 For $\alpha=180^{\circ}$ and inverted mass order, the effective Neutrino Mass for different value of Majorona phases

\begin{tabular}{lll}
\hline$\beta$ & $\gamma$ & $m_{e e}(\mathrm{eV})$ \\
\hline $0^{\circ}$ & $0^{\circ}$ & 0.8171 \\
$0^{\circ}$ & $45^{\circ}$ & 0.8045 \\
$0^{\circ}$ & $90^{\circ}$ & 0.7731 \\
$0^{\circ}$ & $135^{\circ}$ & 0.7404 \\
$0^{\circ}$ & $180^{\circ}$ & 0.7265 \\
$45^{\circ}$ & $0^{\circ}$ & 0.8009 \\
$45^{\circ}$ & $45^{\circ}$ & 0.7899 \\
$45^{\circ}$ & $90^{\circ}$ & 0.7595 \\
$45^{\circ}$ & $135^{\circ}$ & 0.7262 \\
$45^{\circ}$ & $180^{\circ}$ & 0.7104 \\
$90^{\circ}$ & $0^{\circ}$ & 0.7603 \\
$90^{\circ}$ & $45^{\circ}$ & 0.7502 \\
$90^{\circ}$ & $90^{\circ}$ & 0.7202 \\
$90^{\circ}$ & $135^{\circ}$ & 0.6867 \\
$90^{\circ}$ & $180^{\circ}$ & 0.6699 \\
$135^{\circ}$ & $0^{\circ}$ & 0.7173 \\
$135^{\circ}$ & $45^{\circ}$ & 0.7067 \\
$135^{\circ}$ & $90^{\circ}$ & 0.6764 \\
$135^{\circ}$ & $135^{\circ}$ & 0.6430 \\
$135^{\circ}$ & $180^{\circ}$ & 0.6269 \\
$180^{\circ}$ & $0^{\circ}$ & 0.6988 \\
$180^{\circ}$ & $45^{\circ}$ & 0.6863 \\
$180^{\circ}$ & $90^{\circ}$ & 0.6550 \\
$180^{\circ}$ & $135^{\circ}$ & 0.6222 \\
$180^{\circ}$ & $180^{\circ}$ & 0.6081 \\
\hline & & \\
\hline & & \\
\hline & & \\
\hline & & \\
\hline & & \\
\hline
\end{tabular}

In this paper, we have explored the impact of a majorana phases $\alpha, \beta$ and $\gamma$ on the effective neutrino masses. Taking the best-fit value $\Delta_{41}=1.7 \mathrm{eV} \mathrm{V}^{2}$ and $\sin ^{2} \theta_{14}=0.019$. We find the effective neutrino mass $m_{e e}$, for normal neutrino mass order is $m_{e e}^{\text {upper-limit }}=$ $2.0074 \mathrm{eV}$ for $\alpha=0^{\circ}, \beta=0^{\circ}$ and $\gamma=0^{\circ}$ and $m_{e e}^{\text {lower-limit }}=0.6083 \mathrm{eV}$ for $\alpha=$ $180^{\circ}, \beta=180^{\circ}$ and $\gamma=180^{\circ}$. The effective neutrino mass $m_{e e}$, for inverted neutrino mass order is $m_{e e}^{\text {upper-limit }}=2.0069 \mathrm{eV}$ for $\alpha=0^{\circ}, \beta=0^{\circ}$ and $\gamma=0^{\circ}$ and $m_{e e}^{\text {lower-limit }}=0.6081 \mathrm{eV}$ for $\alpha=180^{\circ}, \beta=180^{\circ}$ and $\gamma=180^{\circ}$. We have found due to nonzero value of majorona phases, effective neutrino masses supresses.

Open Access This article is licensed under a Creative Commons Attribution 4.0 International License, which permits use, sharing, adaptation, distribution and reproduction in any medium or format, as long as you give appropriate credit to the original author(s) and the source, provide a link to the Creative Commons licence, and indicate if changes were made. The images or other third party material in this article are included in the article's Creative Commons licence, unless indicated otherwise in a credit line to the material. If material is not included in the article's Creative Commons licence and your intended use is not permitted by statutory regulation or exceeds the permitted use, you will need to obtain permission directly from the copyright holder. To view a copy of this licence, visit http://creativecommonshorg/licenses/by/4.0/. 


\section{References}

1. Super Kamiokande Collaboration, Wendell, R., et al.: Phys. Rev. D 81, 092004 (2010)

2. Super-Kamiokande Collaboration, Ashie, Y., et al.: Phys. Rev. D 71, 112005 (2005)

3. Super-Kamiokande Collaboration, Fukuda, Y., et al.: Phys. Rev. Lett. 82, 1810 (1999)

4. Super-Kamiokande Collaboration, Fukuda, Y., et al.: Phys. Rev. Lett. 82, 2430 (1999)

5. Super-Kamiokande Collaboration, Fukuda, Y., et al.: Phys. Rev. Lett. 82, 2644 (1999)

6. Super-Kamiokande Collaboration, Fukuda, Y., et al.: Phys. Rev. lett. 81, 1562 (1998)

7. Macro Collaboration, Surdo, A., et al.: Nucl. Phys. Proc. Suppl. 110, 342 (2002)

8. Soudan Collaboration, Sanchez, M., et al.: Phys. Rev. D 68, 113004 (2003)

9. Super Kamiokande Collaboration, Cravens, J.P., et al.: Phys. Rev.D 78, 032002 (2008)

10. Abe, K. et al.: arXiv:hep-ex/1010.0118

11. Cleveland, B.T. et al.: Astrophys. J 496, 505 (1998)

12. Cattadori, C., Ferrari, N., Pandola, L.: Nucl. Phys. B (Proc. Suppl.) 143, 3 (2005)

13. Super-Kamiokande Collaboration, Fukuda, Y., et al.: Phys. Lett. B539, 179 (2002)

14. Super-Kamiokande Collaboration, Fukuda, Y., et al.: Phys. Rev. D 73, 112001 (2006)

15. SNO Collaboration, Ahmad, Q.R., et al.: Phys. Rev. Lett. 89, 011301 (2002)

16. Gando, A. et al.: arXiv:hep-ex/1009.4771

17. KamLand Collaboration, Eguchi, K., et al.: Phys. Rev. Lett. 90, 021802 (2003). arXiv:hep-ex/0212021

18. KamLand Collaboration, Araki, T., et al.: Phys. Rev. Lett. 94, 081801 (2005)

19. K2K Collaboration, Aliu, E., et al.: Phy. Rev. Lett. 94, 081802 (2005)

20. Super-Kamiokande Collaboration, Ashie, Y., et al.: Phys. Rev. D 71, 112005 (2005)

21. Freund, M., Hunder, P., Lindner, M.: Nucl. Phys. B 615, 331-357 (2001)

22. Cervera, A. et al.: Nucl. Phy. B 579, 17 (2000)

23. Super-Kamiokande Collaboration, Fukuda, Y., et al.: Phys. Rev. Lett 81, 1562 (1998)

24. Hampel, W.: Phys. Lett. B 447, 127 (1999)

25. CHOOZ Collaboration, Apollonio, C., et al.: Eur. Phy. J 27, 33 (2003)

26. MINOS Collaboration, Habig, A., et al.: Mod. Phys. Lett. A25, 1219 (2010)

27. KamLAND Collaboration, Gando, et al.: arXiv:1009.4771v2

28. Gariazzo, S., et al.: J. Phys. G 43, 033001 (2016)

29. Gariazzo, S., et al.: JHEP 1706, 135 (2017)

30. Barry, J., et al.: JHEP 1107, 091 (2011)

31. Kraus, C., et al.: Eur. Phys. J., 33 (2004)

Publisher's Note Springer Nature remains neutral with regard to jurisdictional claims in published maps and institutional affiliations. 